

\title{
Stability analysis of sampled-data systems using Sum of Squares
}

\author{
Alexandre Seuret, Matthew M. Peet
}

\section{To cite this version:}

Alexandre Seuret, Matthew M. Peet. Stability analysis of sampled-data systems using Sum of Squares. IEEE Transactions on Automatic Control, 2013, 58 (6), pp.1620-1625. 10.1109/TAC.2012.2232378 . hal-00753538

\section{HAL Id: hal-00753538 \\ https://hal.science/hal-00753538}

Submitted on 19 Nov 2012

HAL is a multi-disciplinary open access archive for the deposit and dissemination of scientific research documents, whether they are published or not. The documents may come from teaching and research institutions in France or abroad, or from public or private research centers.
L'archive ouverte pluridisciplinaire HAL, est destinée au dépôt et à la diffusion de documents scientifiques de niveau recherche, publiés ou non, émanant des établissements d'enseignement et de recherche français ou étrangers, des laboratoires publics ou privés. 


\title{
Stability analysis of sampled-data systems using Sum of Squares
}

\author{
Alexandre Seuret and Matthew M. Peet
}

\begin{abstract}
This article proposes a new approach to stability analysis of linear systems with sampled-data inputs or channels. The method, based on a variation of the discrete-time Lyapunov approach, provides stability conditions using functional variables subject to convex constraints. These stability conditions can be solved using the sum of squares methodology with little or no conservatism in both the case of synchronous and asynchronous sampling. Numerical examples are included to show convergence.
\end{abstract}

Index Terms-Sampled-Data systems, Lyapunov function, Sum of Squares.

\section{INTRODUCTION}

In recent years, much attention has been paid to Networked Control Systems (NCS) (see [1], [2]). These systems contain several distributed controllers and plants which are connected through a communication network. In this model, the inputs to the plants are updated as often as the network capacity allows (sampling times) - ideally once per round-trip-time. In such applications, however, a heavy transient load on the network or on a processor can change the sampling period of the controller. To compensate for this effect, the sampling period is sometimes explicitly included in the controller design. However, this approach is problematic in that the sampling period is difficult to predict and variation of this sampling period may destabilize the closed-loop system. The result is that a significant effort has been made to develop stability conditions for sampleddata systems which are robust with respect to variations of sampling period.

Sampled-data systems have been extensively studied in the literature [3]-[7] and the references therein. It is now possible to design controllers which guarantee the robustness of the solutions of the closed-loop system under periodic samplings. However in the case of asynchronous sampling, there are still several open problems. For example, consider the practical situation where the difference between two successive sampling instants is not constant but timevarying. Recently, several articles have addressed the problem of time-varying periods based on a discrete-time approach, [8]-[10]. Recent papers have considered the modeling of continuous-time systems with sampled-data control in the form of continuous-time systems with delayed control input. In [4], a Lyapunov-Krasovskii approach was introduced. Improvements were provided in [5], [11], using the small gain theorem, and in [12], based on the analysis of impulsive systems. These approaches dealt with time-varying sampling periods as well as with uncertain systems (see [4] and [12]). Despite much progress, however, all these conditions are conservative. This means that the sufficient conditions obtained using

A. Seuret is with the CNRS, the Control Systems Department, GIPSA-Lab INP BP 46, Domaine Universitaire - 38400 Saint Martin d'Hères - FRANCE. e-mail: alexandre.seuret@gipsa-lab.genoble-inp.fr. The work of A. Seuret was supported by EU STREP project FeedNetBack FP7-ICT-2007-2.

M.M. Peet is with the Department of Mechanical, Materials, and Aerospace Engineering, Illinois Institute of Technology, Chicago, IL 60616 USA. email: mpeet@iit.edu. This work was partially supported by NSF Grant No. CAREER CMMI 1151018

This paper is partially based on work presented to the IFAC World Congress 2011 continuous time approaches are not able to guarantee asymptotic stability for certain stable systems. Recently several authors [13][15] refined those approaches and obtained tighter conditions.

The key insight of this paper is that once we have developed the discrete-continuous Lyapunov conditions sufficient for stability, then these conditions can be verified computationally using recently developed algorithms for the optimization of polynomial functions. In particular, we use the tools developed in [16] to reformulate the stability question as a convex optimization problem with polynomial variables. We then use the software package SOSTOOLS [17] to solve the optimization problem. As can be seen in the numerical examples, the result is a sequence of stability tests of increasing accuracy. Furthermore, in the numerical examples, the accuracy of the stability test approaches the analytical limit exponentially fast as the degree of the polynomial variables increases.

This article is based on a Lyapunov approach introduced in [18]. This previous result was based on the discrete-time Lyapunov theorem and was expressed using a continuous-time model of sampleddata systems. More precisely, this article analyzed the link between the discrete-time Lyapunov theorem employed, for instance in [8][10], and the continuous-time approach proposed in [4], [12], [13], [15]. In previous work, asymptotic stability criteria can be found for both synchronous and asynchronous samplings. Those criteria were expressed in terms of linear matrix inequalities. The main contribution of this paper is the use of sum of squares tools to construct more sophisticated Lyapunov functions and consequently prove larger upper-bounds for the maximum allowable sampling period than bounds existing in the literature (based on the continuoustime modeling).

This article is organized as follows. The next section formulates the problem. Section III presents a theorem on exponential stability of sampled-data systems expressed in terms of sum of squares. Section IV provides some details on the implementation of the stability conditions using SOSTOOLS. Some examples and simulations are provided in Section $\mathrm{V}$ and show the efficiency of the method. Section VI concludes the article.

Notation : Throughout the article, the sets $\mathbb{N}, \mathbb{R}^{+}, \mathbb{R}^{n}, \mathbb{R}^{n \times n}$ and $\mathbb{S}^{n}$ denote respectively the set of natural numbers, nonnegative real numbers, the set of $n$-dimensional real-valued vectors, the set of $n \times n$ real valued matrices and the subspace of $\mathbb{R}^{n \times n}$ of symmetric matrices. The superscript ' $T$ ' stands for the matrix transposition. The notation $P \succ 0$ for $P \in \mathbb{S}^{n}$ means that $P$ is positive definite. The symbols $I$ and 0 represent the identity and the zero matrices of appropriate dimension. For any matrix $A \in \mathbb{R}^{n \times n}$, the notation $\operatorname{He}(A)$ stands for $A+A^{T}$. We define a functional as any map whose domain includes a function space.

\section{PRoblem Formulation}

Consider the following sampled-data system

$$
\dot{x}(t)=A x(t)+B u\left(t_{k}\right) \quad \forall t \in\left[t_{k}, t_{k+1}\right),
$$

where $x \in \mathbb{R}^{n}$ and $u \in \mathbb{R}^{m}$ represent the state and the input vectors. Define the sampling times $\left\{t_{k}\right\}_{k \in \mathbb{N}}$ to be an increasing sequence of 
positive scalars such that $\bigcup_{k \in \mathbb{N}}\left[t_{k}, t_{k+1}\right)=[0,+\infty)$. Suppose that the sampling intervals, $T_{k}$, are bounded so that there exist positive scalars $T_{\min } \leq T_{\max }$ such that

$$
T_{k}:=t_{k+1}-t_{k} \in\left[T_{\min }, T_{\max }\right], \quad \forall k \in \mathbb{N} .
$$

The sequence $\left\{t_{k}\right\}_{k \in \mathbb{N}}$ represents the sampling instants of the controller. The matrices $A$ and $B$ are constant, known, and of appropriate dimension. The control law is chosen to be linear state feedback,

$$
u=K x
$$

with a gain $K \in \mathbb{R}^{m \times n}$. Then the closed-loop system is governed by

$$
\dot{x}(t)=A x(t)+B K x\left(t_{k}\right) \quad \forall t \in\left[t_{k}, t_{k+1}\right) .
$$

The differential equation (4) with the control law (3) can be integrated over a sampling period. If we define the function

$$
\Gamma(s)=\left[e^{A s}+\int_{0}^{s} e^{A(s-\theta)} d \theta B K\right] \quad \forall s \in\left[0, T_{\max }\right],
$$

we have the following discrete-time system

$$
x\left(t_{k+1}\right)=\Gamma\left(T_{k}\right) x\left(t_{k}\right),
$$

where $x(t)=\Gamma\left(t-t_{k}\right) x\left(t_{k}\right)$ for $t \in\left[t_{k}, t_{k+1}\right)$.

Notation: Taking a cue from time-delay systems theory, we denote the segment of solution on $t \in\left[t_{k}, t_{k+1}\right)$ by $x_{T k}$, so that

$$
x_{T k}(s)=\Gamma(s) x\left(t_{k}\right) \quad \forall s \in\left[0, T_{k}\right) .
$$

We use $\mathscr{K}^{n}$ to denote the space of continuous maps from $\left[0, T_{\max }\right] \rightarrow \mathbb{R}^{n}$, where recall $T_{\max }$ is the upper-bound on the $T_{k}$. This function-based model for sampled-data systems was introduced in [19].

If $T_{k}$ is constant, the discrete dynamics become $x\left(t_{k+1}\right)=$ $\Gamma(T) x\left(t_{k}\right)$, where $T$ is the sampling period. A simple method to check stability of the system is to ensure that $\Gamma(T)$ has all eigenvalues inside the unit circle. If $T_{k}$ is time-varying, then it is not sufficient to verify that $\Gamma\left(T_{k}\right)$ has all eigenvalues inside the unit circle for all $T_{k} \in\left[T_{\min }, T_{\max }\right]$ to prove stability. However, it is still possible to apply the Lyapunov Theorem for discrete-time systems, i.e. find a positive definite matrix $P$ such that $\Gamma^{T}\left(T_{k}\right) P \Gamma\left(T_{k}\right)-P \prec 0$ for all $T_{k} \in\left[T_{\min }, T_{\max }\right]$. This is an infinite dimensional problem in that we must perform an eigenvalue test at an infinite number of points. It is well-known that verifying such infinite-dimensional stability conditions can prove difficult. If the system contains uncertainty in system parameters such as the sampling period, the difficulty increases. Nonetheless, several authors have successfully investigated this approach to stability analysis [8], [9], [20].

Noting that sampled-data systems are a special case of timedelay systems with time-varying delay, many authors treat sampleddata systems in a manner similar to time-delay systems and use similar approaches to the question of stability. For example, sufficient conditions for stability of sampled-data systems were derived in [4] by analyzing stability of a class of systems with time-varying delay. However, these results were somewhat conservative in that they did not account for the unique structure of the variation in delay in a sampled-data system. In [12], the authors introduce a new type of Lyapunov-Krasovskii functional which depends more explicitly on the delay function. In particular, they use the fact the derivative of the delay function representing the effect of the sampling is equal to 1 almost everywhere in their formulation. This led to improvement in the accuracy of the stability conditions. In the present article , we take a different approach which does not model the hold as a delay, but rather uses a new type of sampled-data Lyapunov Theorem introduced in [18] and inspired by [16]. The conditions are enforced using sum of squares optimization.

\section{Stability OF SAMPLED-DATA Systems}

\section{A. Main Theorem}

In this section we introduce a new Lyapunov theorem which applies to general linear sampled-data systems. This theorem accounts for the interaction between the continuous and discrete states of a sampleddata system. A version of this result was introduced in [18] and was partially inspired by the concept of spacing functions introduced in [16]. Essentially, the theorem says that if there exists a Lyapunov function which has a net decrease over every sampling interval, then there exists a storage function which is continuously decreasing for all time. We assume global existence and continuity of solutions.

Theorem 1: [18] For given positive scalars $\alpha, 0<T_{\min } \leq T_{\max }$, suppose $V: \mathbb{R}^{n} \rightarrow \mathbb{R}^{+}$satisfies the following for $0<\mu_{1}<\mu_{2}$

$$
\mu_{1}|x|^{2} \leq V(x) \leq \mu_{2}|x|^{2}, \quad \text { for all } x \in \mathbb{R}^{n} .
$$

Assume that the sampling interval, $T_{k}$, satisfies (2), then the following two statements are equivalent.

(i) There exists a positive constant $\varepsilon$, such that for all solutions $x$ of (4), and for all $k \geq 0$,

$$
V\left(x\left(t_{k+1}\right)\right)-e^{-2 \alpha T_{k}} V\left(x\left(t_{k}\right)\right)<-\varepsilon\left|x\left(t_{k}\right)\right|^{2} .
$$

(ii) There exist a positive constant $\delta$, continuous functionals $Q_{k}: \mathbb{R} \times \mathscr{K}^{n} \rightarrow \mathbb{R}$, differentiable over $\left[t_{k} t_{k+1}\right]$ which satisfy, for all $k \geq 0$ and for all $z \in \mathscr{K}^{n}$

$$
Q_{k}\left(T_{k}, z(\cdot)\right)=e^{-2 \alpha T_{k}} Q_{k}(0, z(\cdot)),
$$

and such that for all solutions of system (4), and for all $t \in\left[t_{k}, t_{k+1}\right]$

$$
\begin{aligned}
& \quad \frac{\mathrm{d}}{\mathrm{d} t}\left[V(x(t))+Q_{k}\left(t-t_{k}, x_{T k}\right)\right] \\
& +2 \alpha V(x(t))+2 \alpha Q_{k}\left(t-t_{k}, x_{T k}\right)<-\delta\left\|x_{T k}\right\| . \\
& \text { where }\left\|x_{T k}\right\|=\max _{\tau \in\left[0, T_{\max }\right]}\left|x_{T k}(\tau)\right| .
\end{aligned}
$$

Moreover, if either of these statements is satisfied, then system (4) is exponentially stable about the origin with a guaranteed decay rate $\alpha$.

Proof: The proof of this theorem can be found in [18].

There are several articles in the literature which use related approaches (see for instance [12], [13]). Typically, these results are derived from a Lyapunov-Krasovskii-type theorem which requires positive definiteness of the functional. In the above result, positivity is relaxed through the use of the spacing functional $Q$.

The following sections show how the conditions of Theorem 1 can be enforced using sum of squares optimization for exponential stability in both the synchronous and asynchronous case. This is similar to the approach taken in [16].

\section{B. Stability under asynchronous sampling}

The case of asynchronous sampling, where $T_{k}$ is unknown but bounded in some range, is clearly more realistic than the synchronous case in a networked control scenario.

$$
\dot{x}(t)=A x(t)+B K x\left(t_{k}\right), \quad \text { for } t \in\left[t_{k}, t_{k}+T_{k}\right), k \geq 0,
$$

where $T_{k}$, for all $k \geq 0$ is a time-varying positive parameter in $\left[T_{\min }, T_{\max }\right]$. The following theorem gives conditions for stability. The conditions of the theorem can be enforced using sum of squares, as will be described shortly.

Theorem 2: Consider System (4). For given $\alpha>0$ and $0<$ $T_{\min }<T_{\max }<\infty$, if there exist $P \in \mathbb{S}^{n}$, positive definite and a bipolynomial matrix $M:\left[0, T_{\max }\right] \times\left[T_{\min }, T_{\max }\right] \rightarrow \mathbb{S}^{2 n}$ such that for 
all $T \in\left[T_{\min }, T_{\max }\right]$,

$$
P \succ 0, \quad\left[\begin{array}{l}
I_{n} \\
I_{n}
\end{array}\right]^{T} M(0, T)\left[\begin{array}{l}
I_{n} \\
I_{n}
\end{array}\right]=0, \quad M(T, T)=0,
$$

and such that for all $\tau \in[0, T]$ and for all $T \in\left[T_{\min }, T_{\max }\right]$, the following inequality holds

$$
\Psi_{\alpha}(\tau, T) \prec 0,
$$

where

$$
\begin{aligned}
\Psi_{\alpha}(\tau, T)= & \Psi(\tau, T)+2 \alpha\left[\begin{array}{c}
0 \\
I_{n}
\end{array}\right] P\left[\begin{array}{c}
0 \\
I_{n}
\end{array}\right]^{T}+2 \alpha M(\tau, T) \\
\Psi(\tau, T)= & \operatorname{He}\left(\left[\begin{array}{c}
0 \\
I_{n}
\end{array}\right] P\left[\begin{array}{c}
(B K)^{T} \\
A^{T}
\end{array}\right]^{T}\right) \\
& +\operatorname{He}\left(M(\tau, T)\left[\begin{array}{cc}
0 & 0 \\
B K & A
\end{array}\right]\right)+\frac{\partial}{\partial \tau} M(\tau, T)
\end{aligned}
$$

Then if $T_{k} \in\left[T_{\min }, T_{\max }\right]$ for all $k \geq 0$, the closed loop system is exponentially stable about the origin with the guaranteed decay rate $\alpha$. Moreover the condition

$$
\Gamma^{T}(T) P \Gamma(T)-e^{-2 \alpha T} P \prec 0
$$

is satisfied for all $T \in\left[T_{\min }, T_{\max }\right]$

Proof: Let $k$ be a positive integer and $T_{k} \in\left[T_{\min }, T_{\max }\right]$. Consider the classical quadratic Lyapunov function for linear continuous-time systems. Define $V: \mathbb{R}^{n} \rightarrow \mathbb{R}^{+}$as $V(x)=x^{T} P x$, where $P \succ 0$ is in $\mathbb{S}$. This function $V$ satisfies condition (6) from Theorem 1. Now define the following function for all $\tau \in\left[0, T_{k}\right]$ and $z \in \mathscr{K}^{n}$

$$
Q\left(\tau, T_{k}, z\right)=\left[\begin{array}{l}
z(0) \\
z(\tau)
\end{array}\right]^{T} M\left(\tau, T_{k}\right)\left[\begin{array}{l}
z(0) \\
z(\tau)
\end{array}\right] .
$$

First, from (10), we note that

$$
\begin{aligned}
Q\left(0, T_{k}, z\right) & =\left[\begin{array}{l}
z(0) \\
z(0)
\end{array}\right]^{T} M\left(0, T_{k}\right)\left[\begin{array}{l}
z(0) \\
z(0)
\end{array}\right] \\
& =z(0)^{T}\left[\begin{array}{l}
I_{n} \\
I_{n}
\end{array}\right]^{T} M\left(0, T_{k}\right)\left[\begin{array}{l}
I_{n} \\
I_{n}
\end{array}\right] z(0) \\
& =0 .
\end{aligned}
$$

Furthermore,

$$
Q\left(T_{k}, T_{k}, z\right)=\left[\begin{array}{c}
z(0) \\
z(T)
\end{array}\right]^{T} M\left(T_{k}, T_{k}\right)\left[\begin{array}{c}
z(0) \\
z(T)
\end{array}\right]=0 .
$$

Therefore, we have $Q\left(T_{k}, T_{k}, z\right)=Q\left(0, T_{k}, z\right)=0$ and hence condition (7) is satisfied.

Computing the derivative term (8), we get

$$
\begin{aligned}
\frac{\mathrm{d}}{\mathrm{d} t} & {\left[V(x(t))+Q\left(t-t_{k}, T_{k}, x_{T k}\right)\right] } \\
& =\dot{x}^{T}(t) P x(t)+x(t)^{T} P \dot{x}(t) \\
& +\frac{\mathrm{d}}{\mathrm{d} t}\left[\left[\begin{array}{c}
x_{T k}(0) \\
x_{T k}\left(t-t_{k}\right)
\end{array}\right]^{T} M\left(t-t_{k}, T_{k}\right)\left[\begin{array}{c}
x_{T k}(0) \\
x_{T k}\left(t-t_{k}\right)
\end{array}\right]\right] \\
& =x^{T}(t) P \dot{x}(t)+\dot{x}^{T}(t) P x(t) \\
& +2\left[\begin{array}{c}
0 \\
\dot{x}(t)
\end{array}\right]^{T} M\left(t-t_{k}, T_{k}\right)\left[\begin{array}{c}
x\left(t_{k}\right) \\
x(t)
\end{array}\right] \\
& +\left[\begin{array}{c}
x\left(t_{k}\right) \\
x(t)
\end{array}\right]^{T} \frac{\mathrm{d}}{\mathrm{d} t} M\left(t-t_{k}, T_{k}\right)\left[\begin{array}{c}
x\left(t_{k}\right) \\
x(t)
\end{array}\right] .
\end{aligned}
$$

Recalling that $\dot{x}(t)=A x(t)+B K x\left(t_{k}\right)$, we get

$$
\begin{aligned}
\frac{\mathrm{d}}{\mathrm{d} t} & {\left[V(x(t))+Q\left(t-t_{k}, T_{k}, x_{T k}\right)\right] } \\
& =2\left[\begin{array}{c}
x\left(t_{k}\right) \\
x(t)
\end{array}\right]^{T}\left(\left[\begin{array}{c}
0 \\
I_{n}
\end{array}\right] P\left[\begin{array}{ll}
B K & A
\end{array}\right]\right)\left[\begin{array}{c}
x\left(t_{k}\right) \\
x(t)
\end{array}\right] \\
& +2\left[\begin{array}{c}
x\left(t_{k}\right) \\
x(t)
\end{array}\right]^{T}\left[\begin{array}{cc}
0 & 0 \\
B K & A
\end{array}\right]^{T} M\left(t-t_{k}, T_{k}\right)\left[\begin{array}{c}
x\left(t_{k}\right) \\
x(t)
\end{array}\right] \\
& +\left[\begin{array}{c}
x\left(t_{k}\right) \\
x(t)
\end{array}\right]^{T} \dot{M}\left(t-t_{k}, T_{k}\right)\left[\begin{array}{c}
x\left(t_{k}\right) \\
x(t)
\end{array}\right] \\
& =\left[\begin{array}{c}
x\left(t_{k}\right) \\
x(t)
\end{array}\right]^{T} \Psi\left(t-t_{k}, T_{k}\right)\left[\begin{array}{c}
x\left(t_{k}\right) \\
x(t)
\end{array}\right],
\end{aligned}
$$

for all $t \in\left[t_{k}, t_{k}+T_{k}\right)$. Introducing the matrix $\Psi_{\alpha}$ in the previous equality yields

$$
\begin{aligned}
& \frac{\mathrm{d}}{\mathrm{d} t}\left[V(x(t))+Q\left(t-t_{k}, T_{k}, x_{T k}\right)\right] \\
& +2 \alpha\left[V(x(t))+Q\left(t-t_{k}, T_{k}, x_{T k}\right)\right] \\
& =\left[\begin{array}{c}
x\left(t_{k}\right) \\
x(t)
\end{array}\right]^{T} \Psi_{\alpha}\left(t-t_{k}, T_{k}\right)\left[\begin{array}{c}
x\left(t_{k}\right) \\
x(t)
\end{array}\right]
\end{aligned}
$$

Thus if there exists a solution of inequality (11), it implies that the left hand side of the previous equality is strictly negative definite. Then integrating this inequality yields, for all $t \in\left[t_{k}, t_{k}+T\right)$

$$
V(x(t))+Q\left(t-t_{k}, T_{k}, x_{T k}\right)<e^{-2 \alpha\left(t-t_{k}\right)} V\left(x\left(t_{k}\right)\right) .
$$

In particular, taking $t=t_{k}+T=t_{k+1}$, we get

$$
V\left(x\left(t_{k+1}\right)\right)<e^{-2 \alpha T_{k}} V\left(x\left(t_{k}\right)\right) .
$$

This ensures exponential stability of the discrete-time system for the constant sampling period $T$. Finally Theorem 1 allows us to conclude the proof.

We emphasize that Theorem 2 only guarantees exponential stability of the solutions of system (9) for any asynchronous sampling which belongs to the interval $\left[T_{\min }, T_{\max }\right]$. If $T_{\min }=T_{\max }=T$, the conditions of Theorem 2 address the problem of exponential stability under constant sampling period $T$.

If $\alpha$ is chosen equal to zero, then the conditions of Theorem 2 concerns now the problem of asymptotic stability under asynchronous samplings characterized by $T_{\min }$ and $T_{\max }$.

As mentioned in the introduction, the case of asymptotic stability of sampled-data systems with a constant sampling period can also be verified numerically by checking if the eigenvalues of the matrix $\Gamma(T)$ lie within the unit circle. However, this method fails if the system matrices $(A, B)$ are uncertain. For example, if matrices $(A, B)$ lie in a polytope, it is difficult to investigate the eigenvalues of the matrix $\Gamma(T)$. By contrast, in Theorem 2, the stability condition depends linearly on the matrices $A$ and $B$. Therefore, it is relatively simple to extend the previous results to the case of systems with polytopic uncertainty. This is a significant advantage of the proposed methodology.

Another remark concerns the choice of the functional $Q$ introduced in Theorem 2. Note that, in (12) in the proof of Theorem 2, the condition $\Psi_{\alpha}(\tau, T)<0$, for all $\tau$ in $[0, T]$ and for all $T \in$ $\left[T_{\min }, T_{\max }\right]$, is equivalent to the negativity of the derivative of the function $V(x(t))+Q\left(t-t_{k}, x_{T k}\right)$. The stability condition $\Psi_{\alpha}(\tau, T)<0$ is equivalent to the negativity of the derivative of the function $V(x(t))+Q\left(t-t_{k}, T_{k}, x_{T k}\right)$. This is not the case in [13] or [18]. Indeed, in these articles, the authors introduce an integral term of the form

$$
\tilde{Q}\left(t-t_{k}, T_{k}, x_{K T}\right)=\left(t_{k+1}-t\right) \int_{t_{k}}^{t} \dot{x}^{T}(s) R \dot{x}(s) \mathrm{d} s,
$$


so that $V+Q+\tilde{Q}$. Although this term has an important role in reducing the conservatism of the stability conditions in [13] and [18], it unavoidably leads to the use of the Jensen's inequality [21] to compute an upper bound of the derivative of $V+Q+\tilde{Q}$. It is well known that the Jensen's inequality introduces conservatism in the stability conditions [22]. In the present paper, this term is unnecessary, yielding an exact condition for the derivative of $V+Q$. This implies that the stability conditions from Theorem 2 should be less conservative than the ones from [13] or [18]. The conservatism of the previous Theorem 2 only depends on the degree, $N$, of the polynomial matrix $M$.

\section{Sum of Squares as Algorithmic Tool}

The conditions associated with Theorem 2 require us to find a matrix $P$ and a function $M(\tau)$ which satisfy linear matrix inequality conditions at an infinite number of values of $\tau$. To test these conditions we must use algorithms for the optimization of functions subject to positivity/negativity constraints. One such algorithm is based on the sum of squares (SOS) decomposition of positive polynomials. To apply this methodology we assume that $M$ is polynomial, can be approximated by a polynomial, or there is a change of coordinates that renders it polynomial.

Denote by $\mathbb{R}[y]$ the ring of polynomials in $y=\left(y_{1}, \ldots, y_{n}\right)$ with real coefficients. Denote by $\Sigma_{s}$ the cone of polynomials that admit a SOS decomposition, i.e., those $p \in \mathbb{R}[y]$ for which there exist $h_{i} \in$ $\mathbb{R}[y], i=1, \ldots, M$ so that

$$
p(y)=\sum_{i=1}^{M} h_{i}^{2}(y) .
$$

If $p \in \Sigma_{s}$, then clearly $p(y) \geq 0$ for all $y$. The converse is not always true, although the converse does hold for univariate matrix-valued polynomials. The advantage of SOS is that while the problem of testing if $p(y) \geq 0$ is known to be NP-hard, testing if $p(y) \in \Sigma_{s}$ is an SDP, [23], and hence is worst-case polynomial-time. The SDPs related to SOS can be formulated and solved efficiently the Matlab toolbox SOSTOOLS [17], which interfaces with semidefinite solvers such as SeDuMi [24].

Consider now the conditions in Theorem 2 . These can be expressed as $L_{1}(0)=L_{2}(T)=0$ with positivity condition

$$
L_{3}(s) \geq 0, \quad s \in \mathscr{S} .
$$

where $L_{1}, L_{2}$ and $L_{3}$ are linear functions of $M$ and $P$ and $\mathscr{S}=$ $[0, T]$. The equality constraints and global positivity can be easily implemented using SOSTOOLS. However, because we only desire positivity on an interval, global positivity may be overly restrictive. To eliminate this conservatism we represent $\mathscr{S}=[0, T]$ as a semialgebraic set:

$$
\mathscr{S}=\left\{s \in R \quad \mid \quad g_{i}(s) \geq 0, \quad i=1, \ldots, M\right\},
$$

where $g_{i}(s)$ are polynomial functions. For $\mathscr{S}=[0, T]$, we use $g_{1}(s, T)=-(T-s) s$. In order to test condition (13), we apply S-procedure-style results which allow us to test positivity on a semialgebraic set using SOS. Specifically, condition (13) holds if there exists SOS polynomials $P_{i} \in \Sigma_{S}$, subject to the constraint

$$
L_{3}(s)+\sum_{i=1}^{M} g_{i}(s) P_{i}(s, y)=P_{0}(s) .
$$

Intuitively, the above condition guarantees that when $s \in \mathscr{S}$, we have $L(s) \leq-\sum_{i=1}^{M} g_{i}(s) p_{i}(s, y) \leq 0$ since $g_{i} \geq 0$ and $p_{i} \geq 0$, and therefore $L(s) \leq 0$ for those $s$. While these conditions are relatively simple, they can, in fact, be made non-conservative through the use of Positivstellensatz results [25].

\begin{tabular}{|c|l|l|}
\hline Theorems & $T_{\max }$ for Ex.1 & $T_{\max }$ for Ex.2 \\
\hline \hline$[4]$ & 0.869 & 0.99 \\
{$[12]$} & 1.113 & 1.99 \\
{$[13]$} & 1.695 & 2.03 \\
{$[14]$} & 1.695 & 2.53 \\
{$[18]$} & 1.723 & 2.62 \\
\hline \hline Th. $2 N=1$ & 0.701 & 2.310 \\
\hline Th. $N=3$ & 1.729 & 3.218 \\
\hline Th. $2 N=5$ & 1.729 & 3.269 \\
\hline
\end{tabular}

TABLE I: Maximum allowable sampling period $T_{\max }$ for examples 1,2 , with $T_{\min }=10^{-6}$.

\begin{tabular}{|c|l|}
\hline Theorems for Ex.3 & $T=T_{\min }=T_{\max } \in\left[\bar{T}_{\min }, \bar{T}_{\max }\right]$ \\
\hline \hline$[18]$ & {$[0.201,1.623]$} \\
\hline Th. $2 N=1$ & $\varnothing$ \\
\hline Th. $2 N=3$ & {$[0.2007,2.016] \bigcup[2.606,3.055]$} \\
\hline Th. $2 N=5$ & {$[0.2007,2.020] \bigcup[2.470,3.694]$} \\
\hline
\end{tabular}

TABLE II: Interval of allowable constant sampling period $T$ for example 3 and $\alpha=10^{-6}$.

a) Application to Theorem 2: For Theorem 2, $M(s, T)$ is a function of two variables $s$ and $T$, where $T \in\left[T_{\min }, T_{\max }\right]$ and $s \in[0, T]$. To describe the relevant semialgebraic set we use

$$
g_{1}(s, T)=-(T-s) s \text { and } g_{2}(T)=-\left(T_{\max }-T\right)\left(T-T_{\min }\right) .
$$

Given these $g_{i}$, we apply the same procedure as for Theorem 2 .

\section{NUMERICAL EXAMPLES}

To illustrate the methodology, we consider control of system (1) with controller $u(t)=K x(t)$ using a network connection where $u(t)$ is updated at times $t_{k}$ where $t_{k+1}-t_{k}=T_{k}$ for all $k=1,2, \cdots, \infty$.

- Example 1 from [4], [12]:

$$
A=\left[\begin{array}{cc}
0 & 1 \\
0 & -0.1
\end{array}\right], B K=\left[\begin{array}{cc}
0 & 0 \\
-0.375 & -1.15
\end{array}\right],
$$

- Example 2 from [13]:

$$
A=\left[\begin{array}{cc}
-2 & 0 \\
0 & -0.9
\end{array}\right], B K=\left[\begin{array}{cc}
-1 & 0 \\
-1 & -1
\end{array}\right],
$$

- and Example 3 from [21], [26]:

$$
A=\left[\begin{array}{cc}
0 & 1 \\
-2 & 0.1
\end{array}\right], B K=\left[\begin{array}{ll}
0 & 0 \\
1 & 0
\end{array}\right] .
$$

Tables I, II and III summarize the results obtained in the literature and using the theorems provided in the present paper for examples 1,2 and 3. One can see that the obtained results are less conservative then existing ones.

In Figures 1, 2 and 3, we use our algorithm to examine the effect of samplings on the decay rate of the system based on the conditions from Theorem 2 applied to synchronous samplings, asynchronous samplings with $T_{\min }=0$ and $T_{\min }=0.9 T_{\max }(\neq 0)$, respectively. Our result shows that for synchronous samplings, the use of an

\begin{tabular}{|c|l|}
\hline Theorems for Ex.3 & {$\left[T_{\min }, T_{\max }\right]$} \\
\hline \hline$[18]$ & {$[0.400,1.251]$} \\
\hline Th.2 $N=1$ & $\varnothing$ \\
\hline Th. $2 N=3$ & {$[0.4,1.820]$ or $[2.680,3.005]$} \\
\hline Th.2 $N=5$ & {$[0.4,1.828]$ or $[2.520,3.550]$} \\
\hline
\end{tabular}

TABLE III: Interval of allowable asynchronous samplings of the form $\left[T_{\min }, T_{\max }\right]$ for example 3 and $\alpha=10^{-6}$. 


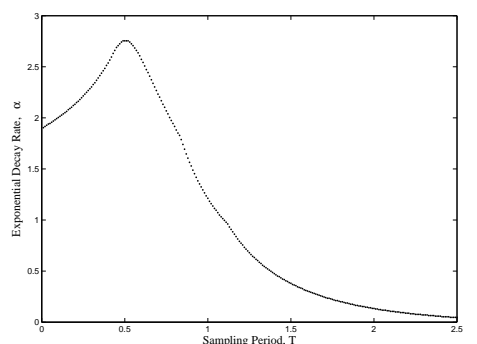

Fig. 1: Bound on Decay Rate vs. Synchronous Sampling Period, $T_{\min }=T_{\max }$ for Example 2 with $N=3$.



Fig. 2: Bound on Decay Rate vs. Asynchronous Sampling Period, $T_{\max }$ and $T_{\min }=0$ for Example 2 with $N=3$.

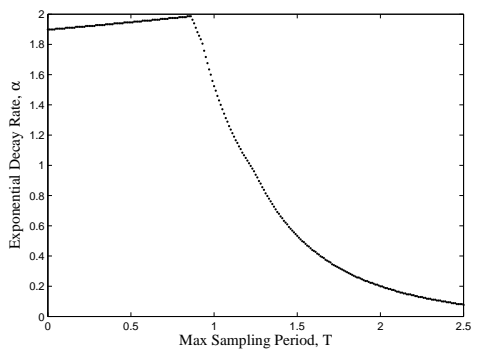

Fig. 3: Bound on Decay Rate vs. Asynchronous Sampling Period, $T_{\max }$ and $T_{\min }=0.9 T_{\max }$ for Example 2 with $N=3$

appropriate sampling period can increase the convergence rate of the same system with a continuous-time controller. This behavior can also be appreciated when one considers asynchronous samplings whose lower bound is strictly greater than 0 as it is shown in Figure 2.

Another important remark deals with Example 3. This system is well known in the time-delay literature because the delay has a stabilizing effect. This means that its solutions are not stable for sufficiently small delay but become stable for sufficiently large delay. The method proposed in this article is able to take into account this phenomena and is also able to isolate several intervals of possible values for the length of the sampling interval where the system is stable for asynchronous and synchronous sampling. Figure 4 illustrates the intervals of stability and exponential decay rate for synchronous sampling.

Note that our analysis of Example 3 indicates that Theorem 2 (with $N=5$ ) can be used to prove stability for asynchronous sampling in $[0.4,1.828]$ OR $[2.520,3.550]$, but not over both simultaneously. This means that stability is not guaranteed if the sampling switches from one interval to the other. This recalls the classical behavior of switched systems: A system which switches between two stable subsystems is not necessarily stable.

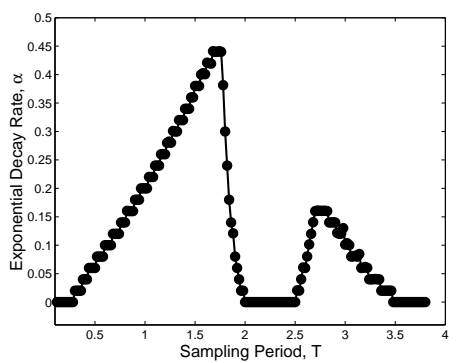

Fig. 4: Bound on Decay Rate vs. Synchronous Sampling Period, $T$ for Example 3 with $N=3$

\section{CONCLUSION}

In this article, a novel analysis of continuous linear systems under asynchronous sampling is provided. This approach is based on the discrete-time Lyapunov Theorem applied to the continuoustime model of the sampled-data systems. Numerical results compare favorably with results in the literature. Perhaps the most important feature of the method presented in this paper is that it is expressed using the sum-of-squares framework and is thus easily extended to nonlinear systems and systems with parametric uncertainty.

\section{REFERENCES}

[1] J. Hespanha, P. Naghshtabrizi, and Y. Xu, "A survey of recent results in networked control systems," Proceedings of the IEEE, vol. 95, no. 1, pp. 138-162, 2007.

[2] S. Zampieri, "A survey of recent results in Networked Control Systems," in Proc. of the $17^{\text {th }}$ IFAC World Congress, Seoul, Korea, July 2008.

[3] T. Chen and B. Francis, Optimal sampled-data control systems. Berlin, Germany: Springer-Verlag, 1995.

[4] E. Fridman, A. Seuret, and J.-P. Richard, "Robust sampled-data stabilization of linear systems: An input delay approach," Automatica, vol. 40, no. 8, pp. 1141-1446, 2004.

[5] H. Fujioka, "Stability analysis of systems with aperiodic sample-andhold devices," Automatica, vol. 45, no. 3, pp. 771-775, 2009.

[6] W. Zhang and M. Branicky, "Stability of networked control systems with time-varying transmission period," in Allerton Conf. Communication, Control, and Computing, October 2001.

[7] W. Zhang, M. Branicky, and S. Phillips, "Stability of networked control systems," IEEE Control Systems Magazine, no. 21, 2001.

[8] Y. Suh, "Stability and stabilization of nonuniform sampling systems," Automatica, vol. 44, no. 12, pp. 3222-3226, 2008.

[9] Y. Oishi and H. Fujioka, "Stability and stabilization of aperiodic sampled-data control systems: An approach using robust linear matrix inequalities," in Joint $48^{\text {th }}$ IEEE Conference on Decision and Control and $28^{\text {th }}$ Chinese Control Conference, December 16-18 2009.

[10] L. Hetel, J. Daafouz, and C. Iung, "Stabilization of arbitrary switched linear systems with unknown time-varying delays," IEEE Trans. on Automatic Control, vol. 51, no. 10, pp. 1668-1674, Oct. 2006.

[11] L. Mirkin, "Some remarks on the use of time-varying delay to model sample-and-hold circuits," IEEE Trans. on Automatic Control, vol. 52, no. 6, pp. 1009-1112, 2007.

[12] P. Naghshtabrizi, J. Hespanha, and A. Teel, "Exponential stability of impulsive systems with application to uncertain sampled-data systems," Systems and Control Letters, vol. 57, no. 5, pp. 378-385, 2008.

[13] E. Fridman, "A refined input delay approach to sampled-data control," Automatica, vol. 46, no. 2, pp. 421-427, 2010.

[14] K. Liu and E. Fridman, "Discontinuous Lyapunov functionals for linear systems with sawtooth delays," in Proc. of the $8^{\text {th }}$ IFAC Workshop on Time-Delay Systems, September 2009.

[15] A. Seuret, "Stability analysis for sampled-data systems with a timevarying period," in Proc. of the $48^{\text {th }}$ IEEE Conference on Decision and Control, December 2009.

[16] M. Peet, A. Papachristodoulou, and S. Lall, "Positive forms and stability of linear time-delay systems," SIAM Journal on Control and Optimization, vol. 47, no. 6, pp. 3227-3258, 2009.

[17] S. Prajna, A. Papachristodoulou, and P. A. Parrilo, "Introducing SOSTOOLS: a general purpose sum of squares programming solver," Proceedings of the IEEE Conference on Decision and Control, 2002. 
[18] A. Seuret, "A novel stability analysis of linear systems under asynchronous samplings," Automatica, vol. 48, no. 1, pp. 177-182, 2012.

[19] Y. Yamamoto, "A function space approach to sampled data control systems and tracking problems," Automatic Control, IEEE Transactions on, vol. 39, no. 4, pp. 703-713, 1994.

[20] L. Hetel, "Robust stability and control of switched linear systems," PhD, Institut National Polytechnique de Lorraine - INPL, Nancy France, 2007.

[21] K. Gu, V.-L. Kharitonov, and J. Chen, Stability of time-delay systems. Birkhauser, 2003.

[22] C. Briat, "Convergence and equivalence results for the Jensen's inequality - application to time-delay and sampled-data systems," IEEE Transactions on Automatic Control, vol. 56(7), pp. 1660-1665, 2011.

[23] P. A. Parrilo, "Structured semidefinite programs and semialgebraic geometry methods in robustness and optimization," Ph.D. dissertation, California Institute of Technology, 2000.

[24] J. F. Sturm, "Using SeDuMi 1.02, a Matlab Toolbox for optimization over symmetric cones," Optimization Methods and Software, vol. 11-12, pp. 625-653, 1999.

[25] M. Putinar, "Positive polynomials on compact semi-algebraic sets," Indiana Univ. Math. J., vol. 42, no. 3, pp. 969-984, 1993.

[26] W. Michiels, S.-I. Niculescu, and L. Moreau, "Using delays and timevarying gains to improve the static output feedback stabilizability of linear systems : a comparison," IMA Journal of Mathematical Control and Information, vol. 21, no. 4, pp. 393-418, 2004. 